\title{
Article \\ A Comparison of Anthropometric and Performance Profiles between Elite and Sub-Elite Hurling Players
}

\author{
John Keane ${ }^{1, *(\mathbb{D}}$, Shane Malone ${ }^{1,2}$, Ciarán Keogh ${ }^{3}$, Damien Young ${ }^{4} \mathbb{D}$, Giuseppe Coratella ${ }^{5}$ \\ and Kieran Collins 1
}

check for updates

Citation: Keane, J.; Malone, S.; Keogh, C.; Young, D.; Coratella, G.; Collins, K. A Comparison of Anthropometric and Performance Profiles between Elite and Sub-Elite Hurling Players. Appl. Sci. 2021, 11, 954. https://doi.org/10.3390/ app11030954

Academic Editor: Mark King Received: 24 December 2020 Accepted: 19 January 2021 Published: 21 January 2021

Publisher's Note: MDPI stays neutral with regard to jurisdictional claims in published maps and institutional affiliations.

Copyright: (c) 2021 by the authors. Licensee MDPI, Basel, Switzerland. This article is an open access article distributed under the terms and conditions of the Creative Commons Attribution (CC BY) license (https:// creativecommons.org/licenses/by/ $4.0 /)$.
1 Gaelic Sport Research Centre, Department of Science, Technological University Dublin, Tallaght, D24 XE62 Dublin, Ireland; shane.malone@mymail.ittdublin.ie (S.M.); kieran.collins@tudublin.ie (K.C.)

2 Research Institute for Sport and Exercise Sciences, Liverpool John Moores University, The Tom Reilly Building, Liverpool L3 3AF, UK

3 Department of Physical Education and Sport Sciences, Health Research Institute, University of Limerick, V94 T9PX Limerick, Ireland; ciaran.keogh@ul.ie

4 Limerick Institute of Technology, Thurles Campus, Thurles, E41 PC92 Tipperary, Ireland; damien.young@lit.ie

5 Department of Biomedical Sciences for Health, Università degli Studi di Milano, 20133 Milan, Italy; giuseppe.coratella@unimi.it

* Correspondence: X00095661@myTUDublin.ie

\begin{abstract}
The aim of the current study was to identify the specific differences in anthropometric and performance profiles between elite and sub-elite hurling players with respect to position and level of play. One hundred and thirty-seven $(n=137)$ hurlers at the elite $(n=61)$ and sub-elite $(n=76)$ level completed a series of anthropometric [height, body mass, the sum of seven skinfolds, adipose tissue percentage estimates (\%AT), fat-free mass estimations (FFM)) and performance ((countermovement jump height (CMJ), CMJ peak power (CMJ PP), CMJ relative peak power (CMJ RPP), acceleration $(5,10$ and $20 \mathrm{~m})$, and yo-yo intermittent recovery test level 1 (Yo-Yo IRT1)) assessments during the "early in-season" phase of the competition. Data were categorized into positions at both levels (full-backs, half-backs, midfielders, half-forwards, full-forwards). Multiple two-way ANOVA's were performed to determine the effect of two fixed factors (level and position) on several anthropometric and performance variables. Elite level players had a significantly lower sum of seven skinfolds across all positions compared to the sub-elite $\left(\eta^{2}=0.441\right.$, large $)$. At half-back, half-forward, and fullforward elite players had significantly lower \%AT $\left(\eta^{2}=0.087-0.167\right.$, small-medium) and greater FFM $\left(\eta^{2}=0.040-0.065\right.$, small). Jump performance assessment showed elite players performed significantly better across all positions for CMJ $\left(\eta^{2}=0.526\right.$, large $)$ CMJ PP $\left(\eta^{2}=0.385\right.$, large $)$ and CMJ RPP $\left(\eta^{2}=0.520\right.$, large $)$. When Yo-Yo IRT1 was considered, elite players completed an increased distance than the sub-elite across all positions $\left(\eta^{2}=0.526\right.$, large $)$. The current data are the first to show differences in positional anthropometric and performance profiles between standards of play within hurling. Applied practitioners should consider these normative data when implementing training programs to maximize position-specific preparation for competition. Furthermore, these data could improve the considerations regarding the transitioning of players from the sub-elite to the elite level of play.
\end{abstract}

Keywords: team sports; playing standards; kinanthropometry; jump performance; intermittent running capacity

\section{Introduction}

Hurling is a field-based intermittent team sport played with a stick (camán) and ball (sliothar) on pitches of standard size (140 m long $\times 90 \mathrm{~m}$ wide) [1]. It is a high-intensity dynamic team sport where two teams of fifteen players (14 outfield players +1 goalkeeper) compete to accumulate more scores than the opponent. Teams aim to strike the ball both over ( 1 point) and under ( 3 points) the crossbar to score with various other skills such as 
the hook, block and solo, all completed using the stick [2,3]. The ebb and flow of matchplay involve players completing repeated high-intensity sprints that are superimposed on periods of low-speed running in an acyclical pattern following the nature of the game [4]. Additional movement demands within the sport include jumping, landing, body contacts and rapid acceleration and deceleration, intertwined with short sharp changes of direction [2]. The frequency of jumping, sprinting and tackling events combined with the known game demands with respect to high-speed running (HSR) highlight the requirement for players to encompass adequate physical and performance profiles to successfully compete in match-play. Hurling can be categorized into the elite (inter-county) and sub-elite (club) levels. At the elite level, teams compete in the National League in the early stages of the competitive calendar, followed by the respective championship structures [5]. Thirty-five teams compete in the National League played across five divisions $(1,2 \mathrm{~A}, 2 \mathrm{~B}, 3 \mathrm{~A}$ and $3 \mathrm{~B})$. At the end of each year's league campaign, one team is promoted from each division excluding division 1 and one team is relegated from each division excluding division 3B. There are also five tiers of hurling championships at the elite level, with the All-Ireland Hurling Championship, the most renowned [2,4]. In a given season, an elite team may compete in over 15 games depending on the level of success during different competitions. At the sub-elite level, the competition structure is more complicated. Each county is responsible for the regulation of the sub-elite league and championship competitions. For teams who are successful within the county structure, there are provincial and All-Ireland competitions to compete within. Elite level match-play is composed of two $35 \mathrm{~min}$ halves (70 $\mathrm{min}$ ) compared to two $30 \mathrm{~min}$ halves $(60 \mathrm{~min})$ at the sub-elite level. While playing rules are almost identical at both levels, several specific differences define both elite and sub-elite match-play that may lead to disparities in the performance demands and anthropometric characteristics between levels.

Differences exist between levels with respect to competition structure. The elite season commences in November (pre-season) and progresses to August (late in-season), while the sub-elite season typically commences in February (pre-season) and progresses to September (late in-season) [1,2]. As the competitive seasons currently overlap, there are often extended periods where sub-elite championships are interrupted while league competitions continue, as selected players are committed to their respective elite inter-county teams. The increased exposure to the elite level training environments with consistent planning and application of training modalities are considered likely to raise the physical standards of the elite players [6,7]. The typical training schedule at the elite level is five days per week (three pitch; two gyms), where a pitch session is replaced by a competitive game in certain week cycles. At the sub-elite level, training schedules will regularly reflect a four-day pattern (three pitch; one gym) with a competitive game on alternating weekends $[7,8]$. In addition to structural differences, further contextual factors may impact the variation of performance between levels. Elite level backroom teams and performance staff will often consist of a team manager, a skills coach, nutritionist, and sports scientist, among others, to try and prepare the players for competitive success $[7,8]$. The increased level of quality support provision at the elite level in terms of consistent strength and conditioning programming, nutritional support, sport science support and technology application highlight the profound gap in the preparatory practices at both levels. This could potentially result in extensive differences between levels when anthropometric and performance profiles are considered [6,9].

The appreciation of physical and physiological performance demands is linked directly to successful performance outcomes within hurling match-play $[2,4,5,10]$. It is paramount that coaches are aware of the performance profiles within their respective squads when preparing players for competitive match-play. The use of anthropometric and performance testing provides an assessment of these characteristics, while subsequent testing throughout the season further informs with respect to seasonal variations and adaption to training prescription [7,11]. Specific body composition assessments such as skinfold thickness and estimations of adipose tissue (\%AT) are commonly assessed within team environments and are often considered default performance-profiling markers [1,12]. Previous literature 
has suggested $\% \mathrm{AT}$ in excess of $15 \%$ can have a negative performance contribution [13]. Increased adiposity can potentially compromise the efficiency of certain sport-specific movements such as accelerations, sprints, jumps and prolonged running where the repeated acceleration of body mass is opposed by gravity $[1,11]$. Reported adiposity levels of the elite hurlers have decreased in recent years from 18.4\% [14] to 12.7\% [10]. The decrease in adiposity is reflective of the increased professionalization of support teams and the application of preparatory practices similar to professional elite team sports.

Explosive power is considered a pivotal performance characteristic within field-based team sports [15]. Contested aerial duals are frequent in hurling match-play, with the attainment of primary possession of paramount importance $[2,10]$. Previous literature indicated the mean vertical jump performance of hurlers $(56.6 \mathrm{~cm}$ ) was less than Gaelic footballers $(62.2 \mathrm{~cm})$ and Soccer players $(59.7 \mathrm{~cm})$ [16]. The countermovement jump (CMJ) profiles of the elite hurlers were reported as $47.2 \pm 5.1 \mathrm{~cm}$ with a positional trend observed from half-forwards $(50.8 \mathrm{~cm})$ to full-backs $(45.2 \mathrm{~cm})$ [10]. Furthermore, it has been suggested that success within team sport performance is influenced by aerobic and anaerobic profiles of players $[17,18]$. The Yo-Yo IRT1 correlates with high-speed running performance and is also reflective of changes in fitness levels $[19,20]$. Position-specific running profiles have been quantified at the elite level with half-backs (TD: $8516 \pm 801 \mathrm{~m}$; HSR: $1086 \pm 385 \mathrm{~m}$ ), midfielders (TD: $8679 \pm 669 \mathrm{~m}$; HSR: $954 \pm 191 \mathrm{~m}$ ) and half-forwards (TD: $8217 \pm 609 \mathrm{~m}$; HSR: $954 \pm 185 \mathrm{~m}$ ) reporting significantly greater running performance demands compared to full-back and full-forwards [2]. An understanding of the HSR capacity of the sub-elite players extrapolated from Yo-Yo IRT1 scores can aid practitioners with the transitioning of players from the sub-elite to the elite level.

While normative data has been provided at the elite level, there is a distinct lack of comparative benchmark data at the sub-elite level. Furthermore, elite teams often implement an "open panel" mantra with regard to player selection, meaning players can transition from the sub-elite to the elite level at any time during the season. Normative data defining the differences in performance profiles across levels is of benefit to coaches to optimize player preparation when transitioning between levels. Therefore, the aim of this study was to identify the differences in anthropometric and performance characteristics between elite and sub-elite hurlers with respect to position and level of play. It was hypothesized that elite level players would exhibit significantly superior profiles when body composition (sum of seven skinfolds and \%AT), jump performance (CMJ, CMJ peak power, and CMJ relative peak power) and intermittent running performance (Yo-Yo IRT1) were considered, compared to the sub-elite.

\section{Materials and Methods}

\subsection{Experimental Approach to the Problem}

The current investigative study aimed to identify the differences in anthropometric and performance characteristics between elite $(n=61)$ and sub-elite $(n=76)$ hurlers (total $n=137)$. Total participant numbers were comprised of two $(n=2)$ elite inter-county squads and three $(n=3)$ division 1 standard sub-elite club squads. All participants underwent anthropometric assessments (height $(\mathrm{cm})$, body mass $(\mathrm{kg})$, the sum of seven skinfolds $(\mathrm{mm})$, adipose tissue percentage estimates (\%AT), fat-free mass estimations $(\mathrm{kg}))$ and performance ((countermovement jump height (CMJ-cm), CMJ peak power (CMJ PP-W), CMJ relative peak power CMJ RPP W. $\mathrm{kg}^{-1}$ ), acceleration over $5 \mathrm{~m}, 10 \mathrm{~m}$ and $20 \mathrm{~m}(\mathrm{~s})$, and yo-yo intermittent recovery test level 1 (Yo-Yo IRT1 m)). Data collection was completed during the "early in-season" phase (June) of the competitive season for both levels [8]. To reduce the extent of external interference, data collection was completed within an indoor environment $[7,8]$. Data collection was also completed at the same time of day (17:00-21:00 h) to minimize the circadian variation across all teams and participants [21]. Participants were encouraged to refrain from vigorous activity for $24-48 \mathrm{~h}$ prior to testing. Participants at both levels were categorized with respect to the positional line of play for further analysis (full-backs, half-backs, midfielders, half-forwards, full-forwards). 


\subsection{Subjects}

The current investigative study aimed to identify the differences in anthropometric and performance characteristics between elite $(n=61)$ and sub-elite $(n=76)$ hurlers (total $n=137)$. One hundred and thirty-seven $(n=137)$ male hurlers from an elite (age; $25 \pm 4$ years, height; $181.8 \pm 5.4 \mathrm{~cm}$, body mass; $85.4 \pm 6.5 \mathrm{~kg}$ ) and sub-elite (age; $25 \pm 5$ years, height; $182.5 \pm 5.8 \mathrm{~cm}$, body mass; $81.8 \pm 7.2 \mathrm{~kg}$ ) level, participated in the current study. When categorized by position at both levels, the breakdown across groups was: (elite (11 full-backs, 13 half-backs, 10 midfielders, 14 half-forwards, 13 full-forwards)) and (sub-elite (13 fullbacks, 18 half-backs, 14 midfielders, 17 half-forwards, 14 full-forwards)). Following ethical approval, participants were briefed on the purpose and benefits of the research and the requirements associated with the study. Written informed consent and medical declaration were obtained as per local institutions' research ethics guidelines (Technological University Dublin, Tallaght Campus).

\subsection{Anthropometry}

Anthropometric assessments were undertaken following the standards of the International Society for the Advancement of Kinanthropometry (ISAK) [22] prior to performance testing. Height and body mass were measured using a Seca Stadiometer and a digital weighing scale (Seca Instruments Ltd., Hamburg, Germany), respectively. Estimations of adipose tissue mass were established by the measurement of subcutaneous fat tissue at seven skinfold sites $(\mathrm{mm})$ (triceps, bicep, subscapular, abdominal, supraspinal, front thigh, medial calf) using Harpenden skinfold calipers were completed by a level 2 ISAK qualified tester (Harpenden Instruments Ltd., Harpenden, UK). Calculations of adiposity (\%AT) were completed using the Reilly equation [1] and again reviewed by a level 2 ISAK qualified tester. Fat-free mass $(\mathrm{kg})$ was calculated by subtracting adipose tissue mass $(\mathrm{kg})$ from the overall body mass $(\mathrm{kg})$ of participants. A technical error of measurement for all anthropometric testing was less than $3 \%$, which is an acceptable measurement error [23].

\subsection{Performance}

Vertical jump height measured using a jump assessment system (Optojump, Bolanzo, Italy) was determined using the average value of three CMJ attempts [24]. The CMJ PP and CMJ RPP were calculated using the Sayers equation [25]. Acceleration times over $20 \mathrm{~m}$ were recorded using a timing gate system (Witty Timing System, Microgate, Bolzano, Italy) with separate gates positioned at $5 \mathrm{~m}, 10 \mathrm{~m}$, and $20 \mathrm{~m}$. All participants completed the Yo-Yo IRT1 to assess repeated high-intensity running performance. It is considered a suitable test that involves an incremental increase of high-intensity movement demands and can be implemented at repeated timepoints within a season to assess changes in performance levels [26]. Jump performance and acceleration tests were completed prior to the Yo-Yo IRT1.

\subsection{Statistical Analysis}

Statistical analysis was performed using the Statistical Package for Social Sciences software (SPSS Version 25.0, Chicago, IL, USA). Data are presented as descriptive statistics (means \pm standard deviation (SD), 95\% confidence intervals) unless stated otherwise. Preliminary analysis on the data was carried out to ensure normal distribution within the data (Shapiro-Wilk test). Further analysis was completed to detect major outliers (greater than three standard deviations), which were subsequently removed $(n=4)$. Multiple two-way ANOVA's were performed to determine the effect of two fixed factors (level and position) on each of the anthropometric and performance variables. Scheffé post hoc correction was used to calculate simple main effects when interaction was observed. Partial eta squared $\left(\eta^{2}\right)$ was reported as a measure of effect size and defined as small 0.02-0.12, medium $0.13-0.25$ and large $>0.26$ [27] with statistical significance set at $p<0.05$. 


\section{Results}

\subsection{Anthropometric}

Anthropometric characteristics of the elite and sub-elite hurlers are shown in Table 1. There was a significant effect detected for positions when height $\left(F_{4,127}=3.834 ; p=0.006\right.$; $\eta^{2}=0.108 ;$ small $)$, body mass $\left(F_{4,127}=4.422 ; p=0.002 ; \eta^{2}=0.122 ;\right.$ small $)$, sum of 7 skinfolds $\left(F_{4,127}=3.524 ; p=0.009 ; \eta^{2}=0.100 ;\right.$ small $)$ and FFM $\left(F_{4,127}=4.453 ; p=0.002 ; \eta^{2}=0.123\right.$; medium) was considered. Scheffé post hoc analysis showed that sub-elite midfielders had significantly lower sum of seven skinfolds compared to half-backs $(p<0.001$; mean difference: $-24.9 \mathrm{~mm})$, half-forwards $(p=0.011$; mean difference: $-18.9 \mathrm{~mm})$ and fullforwards ( $p<0.035$; mean difference: $-17.6 \mathrm{~mm}$ ). Similarly, full-backs were significantly lower than half-backs also $(p=0.007$; mean difference: $-19.9 \mathrm{~mm})$. Sub-elite midfielders were shown to have a significantly lower $\%$ AT than half-backs $(p=0.038$; mean difference: $-1.9 \%$ ) and significantly higher FFM than half-forwards ( $p=0.008$; mean difference: $7.7 \mathrm{~kg}$ ). At elite level, there was no significant effect across positions for any of the anthropometric characteristics $(p>0.05)$.

When comparing anthropometric characteristics at each position between levels of play, significant effects were determined for body mass $\left(F_{1,127}=9.396 ; p=0.003\right.$; $\eta^{2}=0.069 ;$ small $)$, sum of seven skinfolds $\left(F_{1,127}=100.227 ; p<0.001 ; \eta^{2}=0.441\right.$; large $)$, $\%$ AT $\left(F_{1,127}=48.838 ; p<0.001 ; \eta^{2}=0.278 ;\right.$ large $)$, FFM $\left(F_{1,127}=20.957 ; p<0.001 ; \eta^{2}=0.142\right.$; medium), but not for height $\left(F_{1,127}=0.689 ; p=0.408 ; \eta^{2}=0.005 ;\right.$ small $)$. Elite half-backs were significantly heavier than their sub-elite counterparts $(p=0.042$; mean difference: $5.0 \mathrm{~kg} ; \eta^{2}=0.032$, small). Elite players had significantly lower sum of seven skinfolds at all positions [full-backs $\left(p=0.002\right.$; mean difference: $-20.6 \mathrm{~mm} ; \eta^{2}=0.075$, small), halfbacks ( $p<0.001$; mean difference: $-36.6 \mathrm{~mm} ; \eta^{2}=0.244$, medium), midfielders $(p=0.030$; mean difference: $-14.3 \mathrm{~mm} ; \eta^{2}=0.037$, small), half-forwards $(p<0.001$; mean difference: $-36.1 \mathrm{~mm} ; \eta^{2}=0.242$, medium $)$ and full-forwards $(p<0.001$; mean difference: $-28.6 \mathrm{~mm}$; $\eta^{2}=0.150$, medium)]. Finally, elite players had significantly lower \%AT and significantly higher FFM at half-back (\%AT; $p<0.001$; mean difference: $-2.7 \% ; \eta^{2}=0.120$, small, FFM; $p=0.004$; mean difference: $6.7 \mathrm{~kg} ; \eta^{2}=0.065$, small), half-forward (\%AT; $p<0.001$; mean difference: $-3.2 \% ; \eta^{2}=0.167$, medium, FFM; $p=0.023$; mean difference: $5.1 \mathrm{~kg} ; \eta^{2}=0.040$, small) and full-forward (\%AT; $p=0.001$; mean difference: $-2.4 \% ; \eta^{2}=0.087$, small, FFM; $p=0.026$; mean difference: $5.4 \mathrm{~kg} ; \eta^{2}=0.039$, small) positions. 
Table 1. Anthropometric and performance profiles of hurlers with respect to position and level of play. Data presented as mean \pm standard deviation (SD), 95\% confidence intervals. Within the same level, significantly different from a—full-backs; $b$-half-backs; c-midfield; d-half-forward; e-full-forward. Significant difference between levels at respective positions ${ }^{*}(p<0.05),{ }^{* *}(p<0.001)$

\begin{tabular}{|c|c|c|c|c|c|c|}
\hline $\begin{array}{l}\text { Anthropometric } \\
\text { Characteristics }\end{array}$ & Level & Full-Back & Half-Back & Midfield & Half-Forward & Full-Forward \\
\hline \multirow{2}{*}{ Height $(\mathrm{cm})$} & Elite & $179.6 \pm 3.7(176.4,182.7)$ & $183.7 \pm 5.9(180.8,186.6)$ & $183.5 \pm 4.4(180.2,186.8)$ & $181.8 \pm 6.3(179.0,184.6)$ & $180.7 \pm 5.5(177.8,183.6)$ \\
\hline & Sub-elite & $182.0 \pm 6.4(179.1,184.9) \mathrm{c}$ & $182.0 \pm 4.1(179.6,184.5) \mathrm{c}$ & $187.8 \pm 6.3(185.0,190.6)$ abde & $180.6 \pm 4.8(178.0,183.1) \mathrm{c}$ & $180.7 \pm 5.1(177.9,183.5) \mathrm{c}$ \\
\hline \multirow{2}{*}{ Body mass (kg) } & Elite & $84.1 \pm 6.7(80.1,88.1)$ & $86.2 \pm 4.1(82.6,89.9)^{*}$ & $88.2 \pm 4.4(84.0,92.3)$ & $81.6 \pm 5.8(78.1,85.1)$ & $87.5 \pm 8.6(83.9,91.2)$ \\
\hline & Sub-elite & $80.4 \pm 7.5(76.8,84.1)$ & $81.2 \pm 5.6(78.1,84.3)$ & $85.8 \pm 8.9(82.2,89.3) \mathrm{d}$ & $78.7 \pm 6.6(75.5,81.9) \mathrm{c}$ & $83.7 \pm 6.6(80.2,87.3)$ \\
\hline \multirow{2}{*}{ Sum of 7 skinfolds (mm) } & Elite & $50.7 \pm 8.7(41.4,60.1) *$ & $54.6 \pm 16.0(46.0,63.2)^{* *}$ & $52.1 \pm 8.3(42.2,61.9) *$ & $49.1 \pm 8.3(40.8,57.4)^{* *}$ & $55.4 \pm 18.4(46.7,64.0)^{* *}$ \\
\hline & Sub-elite & $71.3 \pm 15.0(62.7,80.0) b$ & $91.2 \pm 25.4(83.9,98.5)$ ac & $66.3 \pm 13.1(58.0,74.6)$ bde & $85.2 \pm 15.2(77.7,92.7) \mathrm{c}$ & $84.0 \pm 13.4(75.7,92.3) \mathrm{c}$ \\
\hline \multirow{2}{*}{ Adiposity (\%AT) } & Elite & $9.7 \pm 1.0(8.6,10.7)$ & $10.0 \pm 2.3(9.0,11.0)^{* *}$ & $9.8 \pm 1.2(8.7,10.9)$ & $9.3 \pm 1.6(8.4,10.2) * *$ & $9.8 \pm 1.8(8.9,10.8)^{* *}$ \\
\hline & Sub-elite & $11.1 \pm 1.4(10.1,12.0)$ & $12.7 \pm 2.7(11.9,13.5) \mathrm{c}$ & $10.8 \pm 1.4(9.9 .11 .8) b$ & $12.5 \pm 1.8(11.7,13.4)$ & $12.2 \pm 1.1(11.3,13.1)$ \\
\hline \multirow{2}{*}{ Fat-free mass $(\mathrm{kg})$} & Elite & $75.9 \pm 5.6(72.2,79.6)$ & $77.6 \pm 4.5(74.2,81.0)$ * & $79.5 \pm 4.2(75.7,83.4)$ & $74.0 \pm 5.4(70.7,77.3)^{*}$ & $79.0 \pm 8.1(75.6,82.4)^{*}$ \\
\hline & Sub-elite & $71.5 \pm 6.5(68.1,74.9)$ & $70.9 \pm 5.3(68.0,73.8)$ & $76.6 \pm 8.7(73.3,79.8) d$ & $68.9 \pm 6.0(65.9,71.8) \mathrm{c}$ & $73.6 \pm 6.0(70.3,76.8)$ \\
\hline \multicolumn{7}{|l|}{$\begin{array}{c}\text { Performance } \\
\text { Characteristics }\end{array}$} \\
\hline \multirow{2}{*}{ CMJ height $(\mathrm{cm})$} & Elite & $45.1 \pm 5.1(41.0,49.2)^{* *}$ & $43.5 \pm 4.0(39.8,47.3)^{* *}$ & $45.0 \pm 3.7(40.7,49.3)^{* *}$ & $42.2 \pm 4.4(38.6,45.8)^{* *}$ & $43.6 \pm 7.8(39.9,47.4) * *$ \\
\hline & Sub-elite & $27.9 \pm 7.8(24.2,31.7)$ & $31.3 \pm 6.1(28.1,34.5)$ & $30.2 \pm 9.7(26.6,33.9)$ & $29.7 \pm 7.5(26.4,32.9)$ & $30.0 \pm 8.4(26.4,33.7)$ \\
\hline \multirow{2}{*}{ CMJ PP (W) } & Elite & $4492 \pm 416(4197,4787)^{* *}$ & $4494 \pm 270(4222,4765)^{* *}$ & $4673 \pm 370(4363,4983)^{* *}$ & $4199 \pm 267(3937,4460)^{* *}$ & $4559 \pm 753(4287,4830)^{* *}$ \\
\hline & Sub-elite & $3284 \pm 535(3012,3555)$ & $3524 \pm 386(3293,3755)$ & $3666 \pm 654(3404,3928)$ & $3311 \pm 552(3073,3548)$ & $3561 \pm 490(3299,3823)$ \\
\hline \multirow{2}{*}{ CMJ RPP $\left(\mathrm{W} \cdot \mathrm{kg}^{-1}\right)$} & Elite & $53.5 \pm 3.8(50.5,56.4) * *$ & $52.2 \pm 2.9(49.4,54.9)^{* *}$ & $53.0 \pm 2.4(49.9,56.1)^{* *}$ & $51.6 \pm 3.5(49.0,54.2) * *$ & $51.9 \pm 5.4(49.2,54.6)^{* *}$ \\
\hline & Sub-elite & $40.9 \pm 5.7(38.2,43.6)$ & $43.4 \pm 4.4(41.1,45.7)$ & $42.8 \pm 6.5(40.2,45.4)$ & $42.0 \pm 6.0(39.6,44.4)$ & $42.7 \pm 5.9(40.0,46.3)$ \\
\hline \multirow[b]{2}{*}{ Acceleration-5 m (s) } & Elite & $1.10 \pm 0.06(0.96,1.23)$ & $1.09 \pm 0.04(0.97,1.21)^{*}$ & $1.11 \pm 0.09(0.96,1.25)$ & $1.08 \pm 0.09(0.96,1.20)$ & $1.11 \pm 0.11(0.98,1.23)$ \\
\hline & Sub-elite & $1.21 \pm 0.30(1.08,1.33)$ & $1.29 \pm 0.32(1.18,1.39)$ & $1.19 \pm 0.32(1.07,1.31)$ & $1.14 \pm 0.25(1.03,1.25)$ & $1.22 \pm 0.29(1.10,1.34)$ \\
\hline \multirow{2}{*}{ Acceleration-10 m (s) } & Elite & $1.81 \pm 0.05(1.67,1.95)$ & $1.83 \pm 0.07(1.70,1.96)^{*}$ & $1.84 \pm 0.08(1.69,1.98)$ & $1.82 \pm 0.05(1.70,1.95)$ & $1.84 \pm 0.05(1.71,1.96)$ \\
\hline & Sub-elite & $1.93 \pm 0.30(1.80,2.06)$ & $2.02 \pm 0.32(1.91,2.13)$ & $1.91 \pm 0.32(1.79,2.04)$ & $1.91 \pm 0.28(1.79,2.02)$ & $1.95 \pm 0.33(1.82,2.07)$ \\
\hline \multirow{2}{*}{ Acceleration-20 m (s) } & Elite & $3.08 \pm 0.12(2.93,3.22)$ & $3.13 \pm 0.08(2.99,3.26)^{*}$ & $3.14 \pm 0.14(2.99,3.29)$ & $3.11 \pm 0.09(2.98,3.24)$ & $3.10 \pm 0.10(2.97,3.23)$ \\
\hline & Sub-elite & $3.20 \pm 0.29(3.06,3.33)$ & $3.34 \pm 0.32(3.22,3.45)$ & $3.18 \pm 0.34(3.05,3.31)$ & $3.19 \pm 0.30(3.07,3.31)$ & $3.20 \pm 0.31(3.07,3.32)$ \\
\hline \multirow[b]{2}{*}{ Yo-Yo IRT1 (m) } & Elite & $2251 \pm 339(2013,2489)^{*}$ & $2305 \pm 308(2085,2524)^{* *}$ & $2588 \pm 174(2338,2838)^{* *}$ & $2286 \pm 364(2075,2497)$ ** & $2234 \pm 293(2015,2453)^{* *}$ \\
\hline & Sub-elite & $1772 \pm 597(1553,1991)$ & $1751 \pm 427(1565,1937)$ & $1854 \pm 461(1643,2065)$ & $1671 \pm 354(1479,1862)$ & $1529 \pm 464(1317,1740)$ \\
\hline
\end{tabular}




\subsection{Performance}

Performance characteristics of the elite and sub-elite hurlers are shown in Table 1. At each respective level, there was no significant difference between individual positions for any of the performance characteristics; CMJ height $\left(F_{4,127}=0.275 ; p=0.894 ; \eta^{2}=0.009\right.$; small $), \operatorname{CMJ~PP~}\left(F_{4,127}=2.832 ; p=0.053 ; \eta^{2}=0.082 ;\right.$ small $), \operatorname{CMJ~RPP~}\left(F_{4,127}=0.236 ; p=0.917 ;\right.$ $\eta^{2}=0.007 ;$ small $), 5 \mathrm{~m}$ acceleration $\left(F_{4,127}=0.463 ; p=0.763 ; \eta^{2}=0.014 ;\right.$ small $), 10 \mathrm{~m}$ acceleration $\left(F_{4,127}=0.362 ; p=0.835 ; \eta^{2}=0.011 ;\right.$ small $), 20 \mathrm{~m}$ acceleration $\left(F_{4,127}=0.690\right.$; $p=0.601 ; \eta^{2}=0.021 ;$ small $)$ and Yo-Yo IRT1 $\left(F_{4,127}=2.383 ; p=0.055 ; \eta^{2}=0.070 ;\right.$ small $)$.

When comparing performance characteristics at each position between levels of play, significant effects were determined for CMJ height $\left(F_{1,127}=140.683 ; p<0.001 ; \eta^{2}=0.526\right.$; large $), \mathrm{CMJ} P \mathrm{PP}\left(F_{1,127}=139.914 ; p<0.001 ; \eta^{2}=0.524 ;\right.$ large $), \mathrm{CMJ} \operatorname{RPP}\left(F_{1,127}=137.418 ;\right.$ $p<0.001 ; \eta^{2}=0.520 ;$ large $), 5 \mathrm{~m}$ acceleration $\left(F_{1,127}=8.158 ; p=0.005 ; \eta^{2}=0.060 ;\right.$ small $), 10 \mathrm{~m}$ acceleration $\left(F_{1,127}=8.126 ; p=0.005 ; \eta^{2}=0.060 ;\right.$ small $), 20 \mathrm{~m}$ acceleration $\left(F_{1,127}=6.612\right.$; $p=0.011 ; \eta^{2}=0.049 ;$ small $)$ and Yo-Yo IRT1 $\left(F_{1,127}=79.592 ; p<0.001 ; \eta^{2}=0.385 ;\right.$ large $)$. Elite level players were shown to have significantly greater jump performance than their sub-elite counterparts across all positions; CMJ height [full-backs $(p<0.001$; mean difference: $17.2 \mathrm{~cm} ; \eta^{2}=0.228$, medium), half-backs $(p<0.001$; mean difference: $12.3 \mathrm{~cm}$; $\eta^{2}=0.160$, medium), midfielders ( $p<0.001$; mean difference: $14.8 \mathrm{~cm} ; \eta^{2}=0.176$, medium), half-forwards ( $p<0.001$; mean difference: $12.6 \mathrm{~cm} ; \eta^{2}=0.169$, medium), and full-forwards $\left(p<0.001\right.$; mean difference: $13.6 \mathrm{~cm} ; \eta^{2}=0.173$, medium $)$ ], CMJ PP [full-backs $(p<0.001$; mean difference: $1208 \mathrm{~W} ; \eta^{2}=0.219$, medium), half-backs $(p<0.001$; mean difference: $969 \mathrm{~W}$; $\eta^{2}=0.186$, medium), midfielders ( $p<0.001$; mean difference: $1007 \mathrm{~W} ; \eta^{2}=0.160$, medium), half-forwards ( $p<0.001$; mean difference: $888 \mathrm{~W} ; \eta^{2}=0.163$, medium) and full-forwards $\left(p<0.001\right.$; mean difference: $998 \mathrm{~W} ; \eta^{2}=0.177$, medium $)$ ], and CMJ RPP [full-backs $(p<0.001$; mean difference: $12.6 \mathrm{~W} \cdot \mathrm{kg}^{-1} ; \eta^{2}=0.233$, medium), half-backs $(p<0.001$; mean difference: $8.7 \mathrm{~W} \cdot \mathrm{kg}^{-1} ; \eta^{2}=0.156$, medium $)$, midfielders $\left(p<0.001\right.$; mean difference: $10.1 \mathrm{~W} \cdot \mathrm{kg}^{-1}$; $\eta^{2}=0.161$, medium $)$, half-forwards $\left(p<0.001\right.$; mean difference: $9.6 \mathrm{~W} \cdot \mathrm{kg}^{-1} ; \eta^{2}=0.185$, medium), and full-forwards ( $p<0.001$; mean difference: $9.2 \mathrm{~W} \cdot \mathrm{kg}^{-1} ; \eta^{2}=0.156$, medium)]. Finally, elite level players at all positions had significantly greater Yo-Yo IRT1 scores compared to their sub-elite counterparts [full-backs $(p=0.004$; mean difference: $479 \mathrm{~m}$; $\eta^{2}=0.063$, small), half-backs $\left(p<0.001\right.$; mean difference: $554 \mathrm{~m} ; \eta^{2}=0.102$, small $)$, midfielders ( $p<0.001$; mean difference: $734 \mathrm{~m} ; \eta^{2}=0.134$, medium $)$, half-forwards $(p<0.001$; mean difference: $615 \mathrm{~m} ; \eta^{2}=0.125$, medium), and full-forwards ( $p<0.001$; mean difference: $705 \mathrm{~m}$; $\eta^{2}=0.142$, medium) ] (Figure 1). 


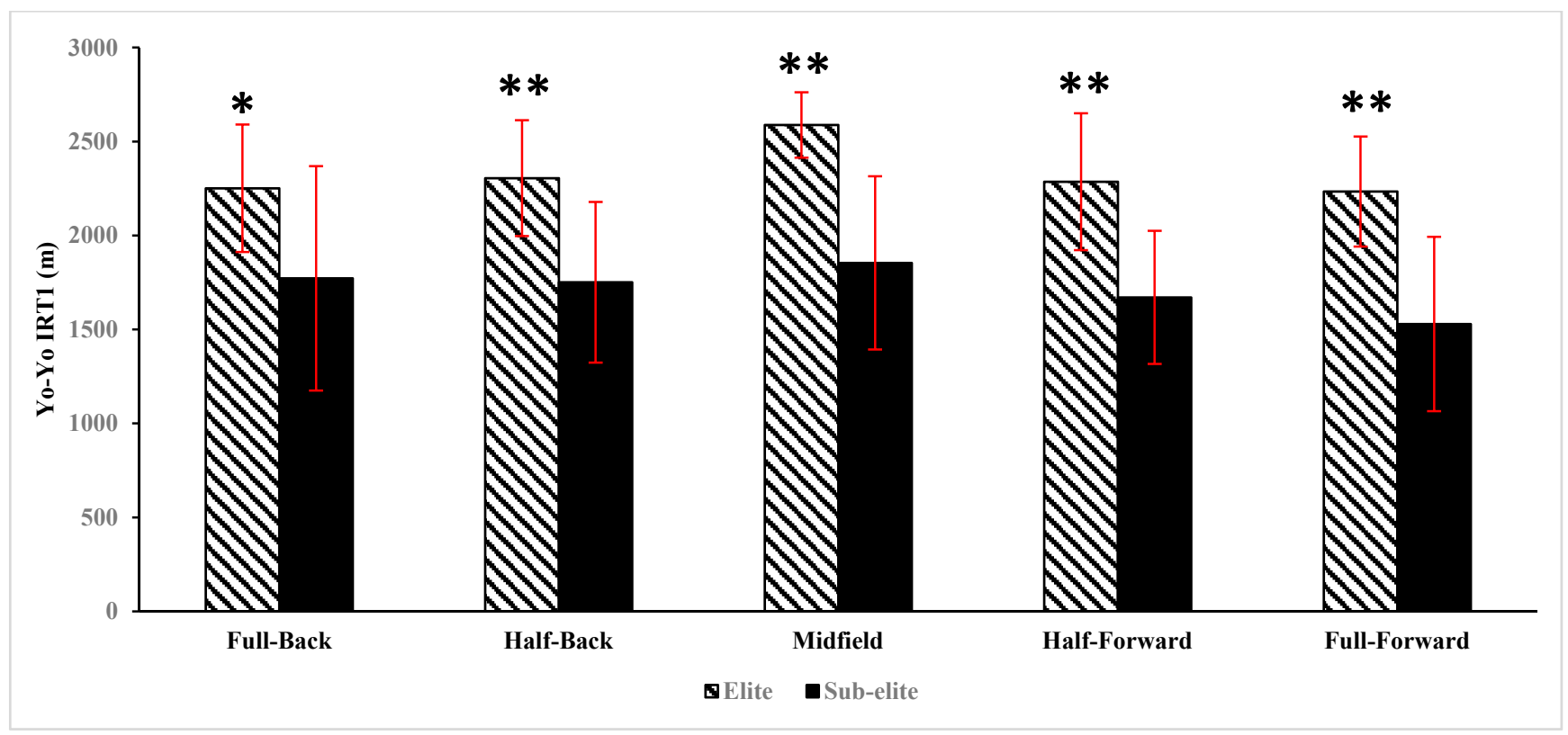

Figure 1. Yo-yo intermittent recovery test level 1 (Yo-Yo IRT1) distance (m) across hurlers with respect to position and level of play. Data presented as mean \pm standard deviation (SD). Significantly greater than sub-elite at respective positions $=*$ $(p<0.05){ }^{* *}(p<0.001)$.

\section{Discussion}

The current study aimed to identify the differences in anthropometric and performance characteristics between elite and sub-elite hurling players with respect to position and level of play. The study is the first to provide a comparative view of the performance profiles across both elite and sub-elite levels of play. The main findings of the investigation show that elite hurlers have significantly greater Yo-Yo IRT1 performance compared to the sub-elite hurlers across all positions. Additionally, across all positions, elite hurlers were shown to have significantly greater jump performance, peak power and relative peak power compared to the sub-elite. Furthermore, at the elite level, players had a significantly lower sum of seven skinfolds across all positions, with the elite hurlers also having a significantly lower \%AT and greater FFM at half-back, half-forward and full-forward compared to the sub-elite. Fewer differences were reported when acceleration profiles were considered, where elite half-backs performed significantly better across $5 \mathrm{~m} .10 \mathrm{~m}$ and $20 \mathrm{~m}$ distances compared to the sub-elite. Finally, elite half-backs were heavier than their sub-elite counterparts while no differences between levels were reported for height. In line with previous work at the elite level, no positional differences were observed across all anthropometric characteristics [10]. In contrast to this work, we observed no significant differences when performance characteristics were considered. The current investigation is the first to provide normative data for performance profiles of the sub-elite level (Table 1).

It is important to consider the differences at an organizational level between elite and sub-elite hurling and how these differences may contribute to the significant variations of anthropometric and performance characteristics. It is widely recognized that at the elite-level preparatory measures for respective squads border professional levels, while at the sub-elite level, an amateur ethos remains inherent $[7,8]$. Backroom and performance staff at the elite level routinely consist of a team manager, several selectors, a skills coach, qualified nutritionist, sports scientist, strength and conditioning coach, and additional support staff to prepare players for competition [7,8]. At the sub-elite level, coherent implementation of strength and conditioning, sports science and nutritional support has yet to be achieved, due in part to the financial disparity between levels [6]. The consistent support of preparatory programming leads to a more robust and powerful athlete [6]. 
At elite-level, this dose of coherent strength training is provided on a consistent training program with players who have represented elite teams for a number of years likely to have a superior baseline training status compared to the typical sub-elite player profile. In contrast, at the sub-elite level, it is considered that training prescription and adaptation are not being maximized to the same extent. Superior jump performance, lower limb power, and acceleration profiles are all evident within this study, with strength and power training a likely contributor to this development. Furthermore, at the elite level, most teams employ a qualified nutritionist to provide educational advice surrounding food intake, hydration strategies and fuel for recovery at a personal and team level. Seasonal monitoring of body composition is common $[28,29]$, with significant efforts to improve anthropometric profiles in relation to post-training food and players' weekly meal considerations applied by team nutritionists. With this increased focus on the nutritional aspect of players' development at the elite level, it was expected that elite hurlers would perform significantly better when anthropometric testing was considered within this study.

Previous research has indicated that elite hurlers are relatively heterogeneous with respect to body size [10]. Within this study, there were minimal differences at the elite level when height and body mass were considered, although the taller players were still centered across transitional lines of the field (half-back and midfield). These positions are still associated with winning primary possession, a key factor when puck-outs and restarts are considered. At the sub-elite level, this heterogenous trend was more pronounced, with midfielders significantly taller than full-backs $(5.8 \mathrm{~cm})$, half-backs $(5.8 \mathrm{~cm})$, halfforwards $(7.2 \mathrm{~cm})$, and full-forwards $(7.1 \mathrm{~cm})$ (all values mean difference). Differences in body mass were minimal across positions. However, it was noticeable that players were heavier, but also leaner at the elite level. Practitioners may consider the manipulation of players' body composition important, as key performance characteristics, including speed, endurance, strength, and power, can be directly influenced by variations in adiposity and lean body mass $[30,31]$. Our data shows that at each independent level, there were further positional differences at the sub-elite level regarding the sum of seven skinfolds, \%AT and FFM. Sub-elite midfielders had significantly reduced skinfolds compared to half-backs $(-24.9 \mathrm{~mm})$, half-forwards $(-18.9 \mathrm{~mm})$ and full-forwards $(-17.6 \mathrm{~mm})$, with full-backs having less than half-backs also $(-19.9 \mathrm{~mm})$. Additionally, sub-elite midfielders had reduced \%AT compared to half-backs $(-1.9 \%)$, while midfielders had greater estimations of FFM than half-forwards $(9.7 \mathrm{~kg})$. Elite level players had a significantly lower sum of seven skinfolds across all five positional lines, with differences ranging from 15-37 mm. At half-back, half-forward, and full-forward elite players decreased \%AT (2.4-3.7\%) and increased FFM (5.1-6.7 kg). The consistent implementation of preparatory programs at the elite level, coupled with increased running performance demands and increased nutritional considerations, may explain the consistent decrease in skinfolds and adiposity while seeing an increase in FFM across elite positions [6,9]. This increase in FFM can reflect positively in terms of muscular density and force production, which can, in turn, result in superior speed, power and jump performance during competitive play $[32,33]$.

Similar to most field-based team sports, possession of the ball (sliothar) is a key determinant to the outcome of the game, as the team in possession has the ability to score while essentially preventing the opposition from scoring. One of the most important aspects of hurling match-play is the ability to win primary possession in contested aerial duals, with the art of "high fielding" considered a particularly important skill to develop [1,2,4]. The decrease in adiposity at the elite level would likely have pre-empted a disparity in lower limb power output due to the enhancement in a player's power to weight ratio [7]. We observed no significant differences across positions at each level individually when CMJ, CMJ PP and CMJ RPP were considered. While not significant, a hierarchical trend was still evident with the transitional line's performance improved. This was in agreement with previous literature for the elite hurlers [10]. Interestingly, CMJ performance was lower than previously reported profiling results at the elite level [10]; this can potentially be explained by an increase in the tactical approach in the game nowadays. Teams are more conscious of 
retaining possession and utilizing short puck-outs and a short passing game, resulting in a reduction of aimless long deliveries where a greater dependency was placed on aerial duals. This has developed into an aim to create space in the inside forward lines where the forwards come onto the ball on the move making it increasingly difficult for backs to spoil possession. Elite players across all positions had significantly greater CMJ, CMJ PP and CMJ RPP compared to the sub-elite. Specifically, ranges of development at the elite level were 12.3-17.2 cm greater CMJ, 888-1208 W greater PP and 8.7-12.6 W $\cdot \mathrm{kg}^{-1}$ greater RPP. This disparity between levels may be attributed to the increase in FFM at the elite level. Additionally, the degree of consistent preparatory support at the elite level will lead to increased strength and power development. There is a considerable effort at the elite level to prioritize the design and structure of training programs that are implemented to improve the strength, power, speed, and endurance capacities of the elite player. A recurring theme is that this level of expertise and application regarding strength training programs is not apparent at the sub-elite level.

Improving the aerobic fitness levels of players is a primary consideration for practitioners given that this component is associated with a reduction in injury risk within intermittent team sports $[34,35]$ and has been correlated with an improved ability to perform repeated sprints and high-intensity actions both with and without possession $[36,37]$. Previous literature has acknowledged the Yo-Yo IRT1 as a reliable assessment of intermittent running capacity within field sports as it correlates with high-intensity running during games and is sensitive to training $[19,20,38]$. Recently, the running demands of the elite and sub-elite match play have been quantified $[2,4,9]$. Positional differences have been presented at the elite level $[2,4]$, while only team average demands have been reported for the sub-elite level to date [9]. At the elite level, half-backs (TD: $8516 \pm 801 \mathrm{~m}$; HSR: $1086 \pm 385 \mathrm{~m}$ ), midfielders (TD: $8679 \pm 669 \mathrm{~m}$; HSR: $954 \pm 191 \mathrm{~m}$ ) and half-forwards (TD: $8217 \pm 609 \mathrm{~m}$; HSR: $954 \pm 185 \mathrm{~m}$ ) were reported to achieve the highest running demands compared to full-back and full-forward [2]. As HSR is considered important during hurling match-play, evaluating the intermittent running capacity of players can identify players suitable for specific playing positions and can also allow coaches to identify players who need to improve this HSR capacity through intervention resulting in a more proficient player. While not statistically significant, we observed a hierarchical trend for intermittent running performance at each level. At both levels, midfielders completed the most distance (elite; $2588 \pm 174 \mathrm{~m}$, sub-elite; $1854 \pm 461 \mathrm{~m}$ ), with the elite half-backs and half-forwards completing more distance than full-backs and full-forwards. Interestingly, at the sub-elite level, full-backs completed the greatest distance after midfielders. When differences between levels were considered, elite level players at all positions completed significantly more distance than sub-elite. At a global level, elite players completed $2322 \pm 322 \mathrm{~m}$ while the sub-elite completed $1715 \pm 459 \mathrm{~m}$. Differences in position between levels ranged from $479 \mathrm{~m}-734 \mathrm{~m}$. Similar to the aforementioned results, there was a significant disparity between levels for Yo-Yo IRT1 performance. While global positioning system (GPS) use is rare at the sub-elite level with only the more financially secure teams able to avail of the technology, at the elite level, the coherent use of GPS technology to quantify training and monitor players is widely recognized. GPS- and running-load prescription allow practitioners to task the elite players with specific loads at varying percentages to work at a high-intensity threshold during selected training drills. This increased exposure to higher running demands in a controlled environment (the use of live GPS systems allows players to attain specific targets) can result in improved tolerance and capacity for highspeed running. This consistent exposure to chronic training demands that are planned and prescribed appropriately allows the elite player to develop running capacity with these advantages not as readily available to the sub-elite.

The current study should not be considered without acknowledgment of specific limitations. The estimation of adiposity, while measured following the standard ISAK protocols, was based on skinfold measurements. This was completed within an acceptable error of measurement and in line with several previous studies [7,8,10,39]; however, 
there nevertheless is the possibility for human error to affect results. In contrast, the use of dual-energy X-ray absorptiometry (DXA) testing is considered the gold-standard measurement for body composition analysis [40]. The current study was not able to include DXA scans due to the associated financial cost. Furthermore, the absence of seasonal variations as a component of this study is also considered a limitation. The practical benefits of providing a benchmark for practitioners for both elite and sub-elite levels have been discussed above. However, the inclusion of seasonal variations would provide analysis on the specific changes that accrue throughout a season following the implementation of varying preparatory programs combined with the implications of competitive match-play as the season progresses from pre to mid to late season. Finally, while the comparative data are beneficial as it can provide a baseline for active practitioners who are currently working with teams to compare their data towards, this study is ultimately comprised of two elite and three sub-elite squads. As such, it can be considered a direct case study analysis of the teams involved. Future research should consider the inclusion of seasonal variations at both levels along with trying to attribute a national-level analysis of hurlers by including participants from an increased number of the elite and sub-elite teams.

\section{Conclusions}

The current study is the first to compare the specific anthropometric and performance profiles of the elite and sub-elite hurling players with respect to position and level of play. The normative data provided for the elite and sub-elite hurlers in the current study can allow practitioners to evaluate their respective squads at each level alongside corresponding baseline profiles. The main findings were that elite hurlers had significantly improved anthropometric and performance characteristics than their sub-elite counterparts. Elite players displayed a superior level of development with a lower sum of seven skinfolds, greater jump performance and Yo-Yo IRT1 performance across all positional lines of play. Additionally, elite half-backs, half-forwards and full-forwards had significantly lower \%AT and greater levels of FFM compared to the sub-elite. Players at the sub-elite level can essentially transition between sub-elite and elite all year based on the understanding of "open panels" for selection. Coaches should consider further implementation of specific testing parameters at the sub-elite level to identify positional profiles, allowing for more accurate and applied planning and prescription of training modalities to improve performance levels. Coaches and practitioners may use these data to understand the significant contrast between levels. The careful and gradual transition from the sub-elite-to-elite level with an improved understanding of the planning and prescribing of training load can potentially modify injury risk as the difference in physical and performance characteristics appears evident. While previous literature has highlighted the disparity in time involved at the elite level regarding the number of weekly sessions, recent insight would indicate that it is not the amount of time being spent preparing teams but the quality of this preparation that should be focused on. The support provision at the elite level with respect to nutritional education, conditioning and application, sport science support with the increased nuance of GPS technology, and performance analysis supersedes the preparation at the sub-elite level. Ultimately this work provides a snapshot of the growing disparity between levels and suggests that the transition of players from the sub-elite to the elite level requires increased planning and consideration to make the transition safe for players while also improving the readiness to perform successfully at the elite level.

Author Contributions: J.K., S.M., D.Y. and K.C. designed the study. Data collection was completed by J.K. and S.M. Data analysis was completed by J.K., S.M., C.K. and K.C. Interpretation and manuscript preparation was conducted by J.K., K.C. and G.C. and supported by all authors. All authors have read and agreed to the published version of the manuscript.

Funding: Research funding Technological University Dublin, Tallaght Campus. JK receives Presidents Research Award, 2018-2020. 
Institutional Review Board Statement: The study was conducted according to the guidelines of the Declaration of Helsinki, and approved by the Institutional Ethics Committee of Technological University Dublin (Approval number; REC-PG4-20718, Date approved; 25 July 2018).

Informed Consent Statement: Informed consent was obtained for this research.

Acknowledgments: The author would like to thank all the players, coaches and teams who participated in this research study.

Conflicts of Interest: The authors declare no conflict of interest regarding this study.

\section{References}

1. Reilly, T.; Collins, K. Science and the Gaelic sports: Gaelic football and hurling. Eur. J. Sport Sci. 2008, 8, 231-240. [CrossRef]

2. Young, D.; Beato, M.; Mourot, L.; Coratella, G. Match-Play Temporal and Position-Specific Physical and Physiological Demands of Senior Hurlers. J. Strength Cond. Res. 2020, 34, 1759-1768. [CrossRef] [PubMed]

3. Malone, S.; Hughes, B.; Reilly, D.K.C.T.; Akubat, I. Methods of Monitoring Training Load and Their Association with Changes Across Fitness Measures in Hurling Players. J. Strength Cond. Res. 2020, 34, 225-234. [CrossRef]

4. Reilly, D.K.C.T.; McRobert, A.; Morton, J.P.; Sullivan, D.O.; Doran, D.A. The Work-Rate of Elite Hurling Match-Play. J. Strength Cond. Res. 2018, 32, 805-811. [CrossRef]

5. Young, D.; Coratella, G.; Malone, S.; Collins, K.; Mourot, L.; Beato, M. The match-play sprint performance of elite senior hurlers during competitive games. PLOS ONE 2019, 14, e0215156. [CrossRef] [PubMed]

6. Mullane, M.; Turner, A.; Bishop, C. Strength and Conditioning Considerations for Hurling. Strength Cond. J. 2018, 40, 72-84. [CrossRef]

7. Kelly, R.A.; Reilly, D.K.C.T. The Seasonal Variations in Anthropometric and Performance Characteristics of Elite Intercounty Gaelic Football Players. J. Strength Cond. Res. 2018, 32, 3466-3473. [CrossRef]

8. Shovlin, A.; Roe, M.; Malone, S.; Reilly, D.K.C.T. Positional Anthropometric and Performance Profile of Elite Gaelic Football Players. J. Strength Cond. Res. 2018, 32, 2356-2362. [CrossRef]

9. Young, D.; Mourot, L.; Coratella, G. Match-play performance comparisons between elite and sub-elite hurling players. Sport Sci. Health 2018, 14, 201-208. [CrossRef]

10. Reilly, D.K.C.T. Anthropometric and Performance Characteristics of Elite Hurling Players. J. Athl. Enhanc. 2014, 3. [CrossRef]

11. Doran, D.A.; Donnelly, J.P.; Reilly, T. Kinanthropometric and performance characteristics of Gaelic games players. In Kinanthropometry VIII; Routledge: London, UK, 2003.

12. Reilly, T.; Doran, D. Science and Gaelic football: A review. J. Sports Sci. 2001, 19, 181-193. [CrossRef] [PubMed]

13. Doran, D.A.; Mc Geever, S.; Collins, K.D.; Quinn, C.; McElhone, R.; Scott, M. The Validity of Commonly Used Adipose Tissue Body Composition Equations Relative to Dual Energy X-ray Absorptiometry (DXA) in Gaelic Games Players. Int. J. Sports Med. 2013, 35, 95-100. [CrossRef] [PubMed]

14. McIntyre, M.C. A comparison of the physiological profiles of elite Gaelic footballers, hurlers, and soccer players. Br. J. Sports Med. 2005, 39, 437-439. [CrossRef] [PubMed]

15. Coneyworth, P.; Ward, N.; Turner, A.N. A field-based fitness testing battery for Rugby League. Prof. Strength Cond. 2012, 25, 4-9.

16. Brick, N.; O'Donoghue, P. Fitness Profiles of Elite Players in Hurling and Three Football Codes: Soccer, Rugby Union and Gaelic Football. In Science and Football V; Araújo, D., Cabri, J., Reilly, T., Eds.; Routledge: London, UK, 2005; p. 172.

17. Malone, S.; Hughes, B.; Reilly, D.K.C.T. The Influence of Exercise-to-Rest Ratios on Physical and Physiological Performance during Hurling-Specific Small-Sided Games. J. Strength Cond. Res. 2019, 33, 180-187. [CrossRef]

18. Thomas, C.; Comfort, P.; Jones, P.A.; Dos'Santos, T. Strength and Conditioning for Netball. Strength Cond. J. 2017, 39, 10-21. [CrossRef]

19. Bangsbo, J.; Iaia, F.M.; Krustrup, P. The Yo-Yo Intermittent Recovery Test. Sports Med. 2008, 38, 37-51. [CrossRef]

20. Deprez, D.; Coutts, A.J.; Lenoir, M.; Fransen, J.; Pion, J.; Philippaerts, R.M.; Vaeyens, R. Reliability and validity of the Yo-Yo intermittent recovery test level 1 in young soccer players. J. Sports Sci. 2014, 32, 903-910. [CrossRef]

21. Reilly, T.; Brooks, G.A. Exercise and the Circadian Variation in Body Temperature Measures. Endoscopy 1986, 7, 358-362. [CrossRef]

22. Marfell-Jones, M.; Stewart, A.; de Ridder, J. International Standards for Anthropometric Assessment; International Society for the Advancement of Kinanthropometry: Wellington, New Zealand, 2012.

23. Atkinson, G.; Reilly, T.; Marfell-Jones, M. What is this thing called measurement error? In Kinathropometry VIII, Proceeding of the 8th International Conference of Kinathropometry, Manchester, UK, 19-23 July 2002; Reilly, T., Marfell-Jones, M., Eds.; Routledge: London, UK, 2013; pp. 3-13.

24. Bosco, C.; Luhtanen, P.; Komi, P. A simple method for measurement of mechanical power in jumping. Eur. J. Appl. Physiol. 1983, 50, 273-282. [CrossRef]

25. Sayers, S.P.; Harackiewicz, D.V.; Harman, E.A.; Frykman, P.N.; Rosenstein, M.T. Cross-validation of three jump power equations. Med. Sci. Sports Exerc. 1999, 31, 572-577. [CrossRef] [PubMed]

26. Malone, S.; Hughes, B.; Reilly, D.K.C.T. Are small-sided games an effective training methodology for improving fitness in hurling players? A comparative study of training methodologies. Int. J. Sports Sci. Coach. 2017, 12, 685-694. [CrossRef] 
27. Cohen, J. Statistical Power Analysis for the Behavioral Sciences, 2nd ed.; Lawrence Erlbaum: Mahwah, NJ, USA, 1988.

28. Davies, R.W.; Toomey, C.M.; Mc Cormack, W.; Hughes, K.; Cremona, A.; Jakeman, P. Body composition analysis of inter-county Gaelic athletic association players measured by dual energy X-ray absorptiometry. J. Sports Sci. 2016, 34, 1015-1020. [CrossRef] [PubMed]

29. Davies, R.W.; Toomey, C.M.; Mc Cormack, W.; O’Neill, C.; Hughes, K.; Jakeman, P. Seasonal changes in body composition of inter-county Gaelic Athletic Association hurlers. J. Sports Sci. 2016, 35, 2427-2432. [CrossRef]

30. Bilsborough, J.C.; Greenway, K.; Livingston, S.; Cordy, J.; Coutts, A.J. Changes in Anthropometry, Upper-Body Strength, and Nutrient Intake in Professional Australian Football Players during a Season. Int. J. Sports Physiol. Perform. 2016, 11, 290-300. [CrossRef]

31. Cullen, B.D.; Cregg, C.J.; Kelly, D.T.; Hughes, S.M.; Daly, P.G.; Moyna, N.M. Fitness Profiling of Elite Level Adolescent Gaelic Football Players. J. Strength Cond. Res. 2013, 27, 2096-2103. [CrossRef]

32. Ackland, T.; Lohman, T.G.; Sundgot-Borgen, J.; Maughan, R.J.; Meyer, N.L.; Stewart, A.D.; Müller, W. Current Status of Body Composition Assessment in Sport. Sports Med. 2012, 42, 227-249. [CrossRef]

33. Suchomel, T.J.; Nimphius, S.; Stone, M.H. The Importance of Muscular Strength in Athletic Performance. Sports Med. 2016, 46, 1419-1449. [CrossRef]

34. Malone, S.; Hughes, B.; Doran, D.A.; Reilly, D.K.C.T.; Gabbett, T.J. Can the workload-injury relationship be moderated by improved strength, speed and repeated-sprint qualities? J. Sci. Med. Sport 2019, 22, 29-34. [CrossRef]

35. Gabbett, T.J. The training-injury prevention paradox: Should athletes be training smarter and harder? Br. J. Sports Med. 2016, 50, 273-280. [CrossRef]

36. Durandt, J.; Tee, J.; Prim, S.K.; Lambert, M. Physical Fitness Components Associated with Performance in a Multiple-Sprint Test. Int. J. Sports Physiol. Perform. 2006, 1, 150-160. [CrossRef] [PubMed]

37. Sirotic, A.C.; Coutts, A.J. Physiological and performance test correlates of prolonged, high-intensity, inter-mittent running performance in moderately trained women team sport athletes. J. Strength Cond. Res. 2007, 21, 138-144. [CrossRef] [PubMed]

38. Veale, J.P.; Pearce, A.J.; Carlson, J.S. The Yo-Yo Intermittent Recovery Test (Level 1) to discriminate elite junior Australian football players. J. Sci. Med. Sport 2010, 13, 329-331. [CrossRef]

39. Muñoz, C.S.; Molina, J.J.M.; Cañas, J.; Courel-Ibáñez, J.; Sánchez-Alcaraz, B.J.; Zabala, M. Anthropometric and Physical Fitness Profiles of World-Class Male Padel Players. Int. J. Environ. Res. Public Health 2020, 17, 508. [CrossRef] [PubMed]

40. Schousboe, J.T.; Shepherd, J.A.; Bilezikian, J.P.; Baim, S. Executive Summary of the 2013 International Society for Clinical Densitometry Position Development Conference on Bone Densitometry. J. Clin. Densitom. 2013, 16, 455-466. [CrossRef] 